Physical Sciences | Matthew Suss \& Eric Guyes

\section{Turning salt water into fresh water with capacitive deionization cycling} Fresh water is one of the key ingredients for sustaining human life. Obtaining sufficient quantities of safe and drinkable water remains a challenge, unusable water through a process known as desalination. Professor Matthew Suss at the Technion Israel Institute of Technology, with his PhD candidates Mr Eric Guyes and Ms Rana Uwayid, are researching one important technique - capacitive deionization - to make the process more efficient.

The Earth's surface might be over $70 \%$ water but less than $3 \%$ of that
is fresh water. Of that $3 \%$, over hal is trapped in the form of glaciers and ce caps, leaving just a small remaining percentage of drinkable ground water While water might seem abundant, the small freshwater reserves on Earth mean that access to this precious resource is precarious. Drinkable water is so scarce that even small increases in demand are enough to cause considerable stress to

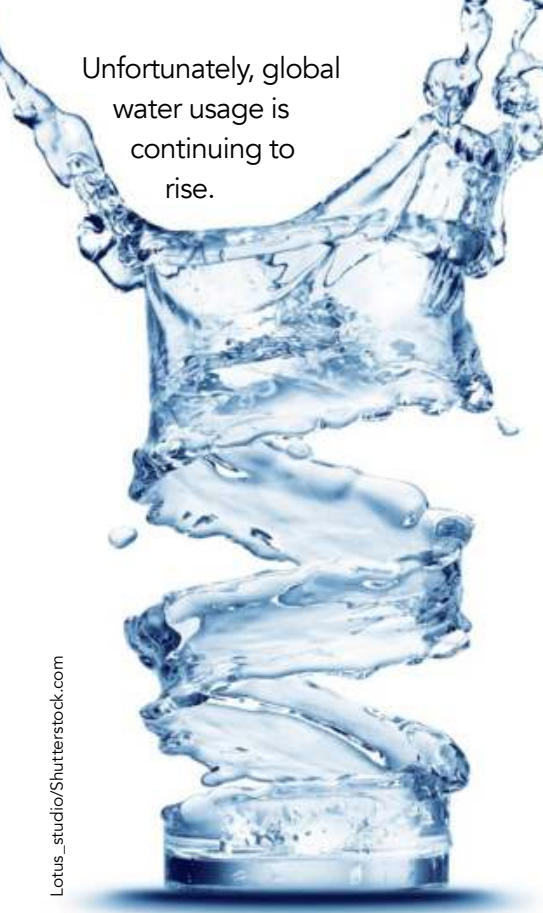

process, by working with a particular (c) cycling. The advantage of this method fdesalination is that it is far more compact and can be tuned to leave Part of the team's work is finding ways to optimise the cell and electrode designs to do just this.

\section{DESALINATION PROCESS}

There are many ways to remove salt from water. One is simply to boil the and then allowing the water vapour to condense again. This is known as thermal distillation and, while simple, is incredibly energy intensive. Instead, most standard desalination plants make use of membranes that filter different chemical species from the water, leaving desalinated water as a product. Destination is the salt from water, and in some senses it

- One of the challenges of this emerging technology is dealing with the problem of electrode degradation.

\section{water crisis on a planet where most of our \\ sually salts in water exist as ions,} water is found in the oceans. Why, then, do desalination plants produce less than $1 \%$ of the world's drinking water? Part complexity of the desalination process, which requires expensive equipment, heavy maintenance, and large plant sites. thalso has a sizeable energy footprint, often making it an unattractive option terms of energy and financial cost.

Dr Matthew Suss, Mr Eric Guyes, and Ms Rana Uwayid at the Technion Israel Institute of Technology are taking a which are charged chemical species. Depending on the design, membranes can be used to filter ions based on their size or charge. One of the problems is oflination plants face is that water is often treated for purficication using before desalinition, and any residual chlorine can cause damage to these expensive constructions.

Capacitive deionization works in a markedly different way to this 'revers the huge advantage of only requiring very low voltages (the voltage demand is less than that produced by a single AAbattery. Capacilve deionization which contains two pie wes of carbo a called the electrodes. A voltage is then applied between them, causing a current to flow and the salt to move into the electrodes, where it can be stored.

What makes capacitive deionization such a remarkable technique is the structure of the electrodes. These are made from nanoporous carbon, meaning that each electrode has a series of miniature tunnels through its structure, making the total surface area more than a thousand square meters the size of five tennis courts - for jus oxpansive tore large quantities of salt and this torage capability can be enhanced even fuld by treating the electrodes with acid.

CORROSIVE CHEMISTRY The potential portability of capacitive deionization and low energy demands make it an attractive alternative to membrane filtration. One of the challenges for progressing this emerging technology, however, is dealing with the problem of electrode degradation.

As a voltage is applied across the electrodes, the carbon structures can start to break cown, hindering their sat Utorage capabilities. Suss, Guyes, and the longevity of the carbon electrodes through two methods. One is simply reducing the time the voltage is applied for. This has the effect of dramatically increasing the stability of the carbon and the electrode lifetime.

The other approach is to change the construction of the electrode. Suss and Uwayid found that introducing multiple stacked layers of carbons also significanty enhanced electrode lifetimes. Their work in this area not only involves developing new electrodes, but attempting to fully understand the chemistry that is part of the degradation process. Understanding voltare cyling process is key to bect altage cycling process is key to being

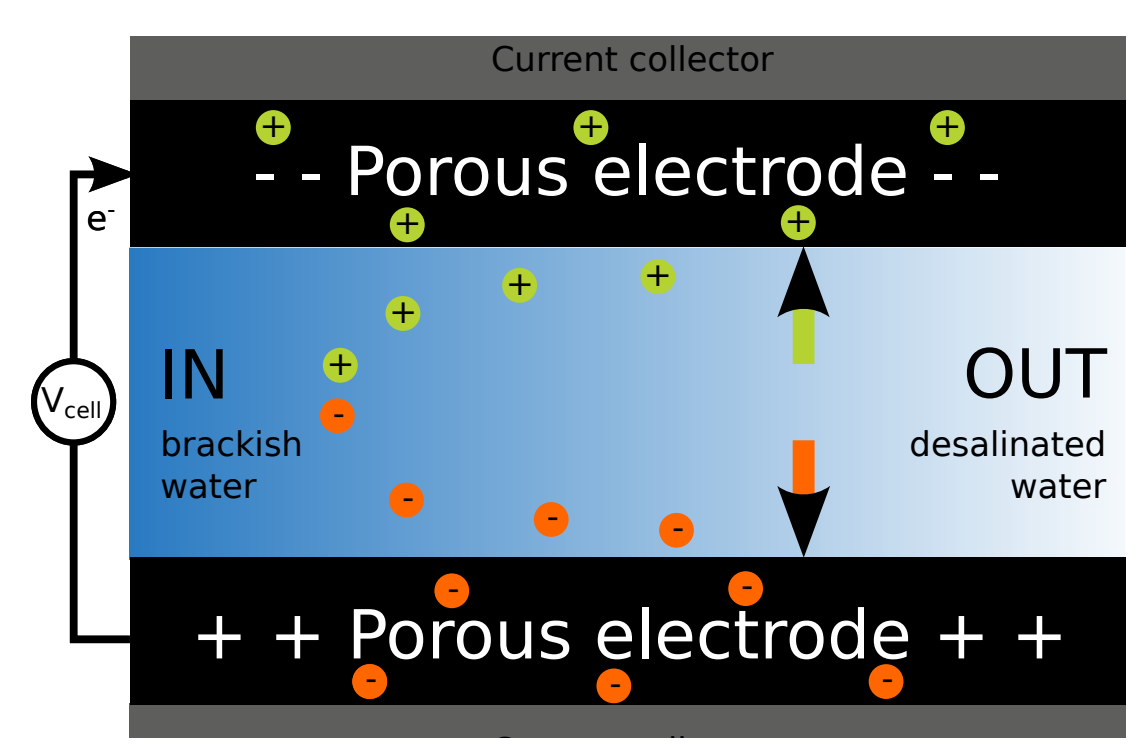

Capacitive deionization works by pumping the water into a cell containing two pieces of carbon (the
electrodess. A voltage is then applied between them, causing a current to fllow and the salt to move into
the electrodes, where it can be

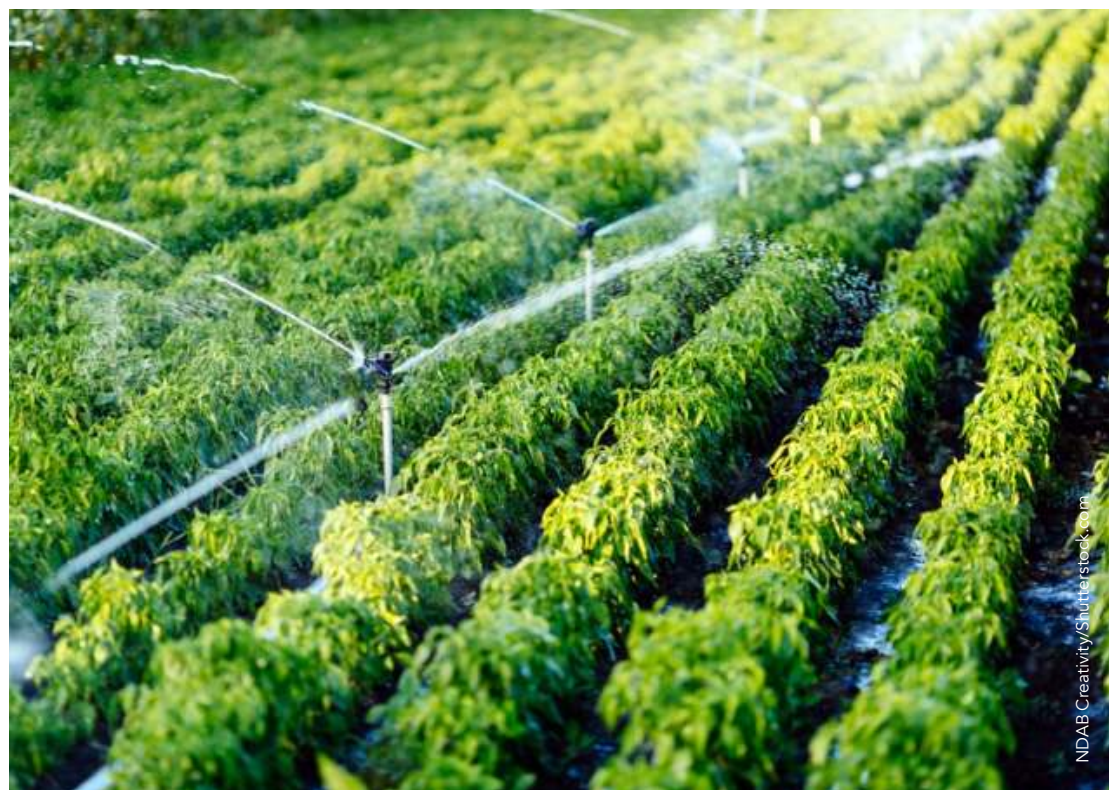
Capacitive deionization can
crop health and production.

and improve the performance of the technology. The team believes their recent improvements in cell stabilities are vital for being able to develop exceptionally long-lasting, highly and systems for wat capacitive deionization

SELECTIVE FILTERING

Capacitive deionization cells do not just convert salt water into fresh. Suss and Guyes have managed to change the carbon electrode design to create sertive fleer for df ferentionic species. Freshwater for inigation must have a low is less problem and therefore removal of sodium species must instead be prioritised. By electrodes, Suss and Guyes have been able to achieve excellent sodium/ca that maintains this performance for over

As the demand for water grows The alogy in providng dinkable water. The ability to build cells on both small and large scales may also make this a considerably more flexible solution than current water desallnation plants that separation factors and create a stable cell capacitive deionization may be a key 


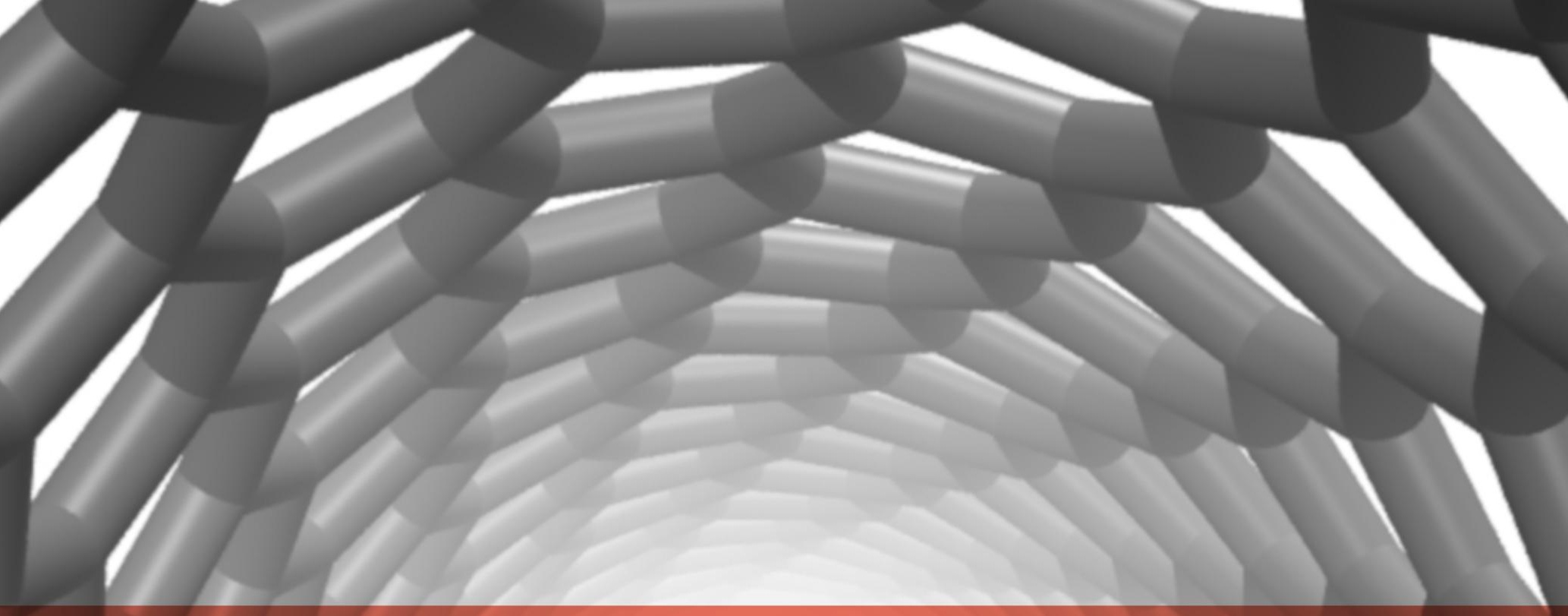

